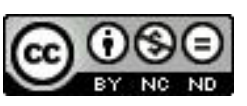

\title{
Marco Figural como Medio para Factorizar Polinomios Cuadráticos
}

\section{Marco Figural how to factorize tools Polynomials Quadratics}

Teresita Méndez Olave*

\begin{abstract}
Resumen
Este artículo es una propuesta para la enseñanza - aprendizaje de la factorización de polinomios cuadráticos, sustentado en los trabajos de Regine Douady sobre el juego de marcos de su teoría Dialéctica Herramienta Objeto, ella destaca el rol de la articulación de marcos matemáticos como un medio para acceder a "formulaciones diferentes de un problema que sin ser necesariamente equivalentes permiten un nuevo acercamiento a las dificultades encontradas y la puesta en escena de útiles y técnicas que no se impusieron en la primera formulación". Bajo esta perspectiva se construye un sistema axiomático, en un marco figural en el que se definen objetos primarios, y se establecen relaciones entre ellos que van dando paso a definiciones y teoremas de una teoría matemática en este marco, proponiendo reglas según las que se operaran los objetos y un juego que permite operacionalizar la factorización. Este marco se convierte en vehículo para comprender las difíciles relaciones de los objetos de la factorización en el álgebra. Esta forma de trabajo didáctico desarrolla habilidades cognitivas y metacognitivas en los estudiantes, quienes descubren y enuncian propiedades de la factorización, establecen las relaciones algebraicas entre los coeficientes del polinomio y los factores que factorizan la expresión, desarrollando una actividad matemática.
\end{abstract}

Palabras-clave: Factorización. Marco Figural. Categorías de la Factorización. Juego de Marcos.

\footnotetext{
* Magister enseñanza de las ciencias c/m didáctica de la matemática, Universidad Católica de Valparaíso/UCV, Chile. Académica del Departamento de Formación Inicial y Escolar de la Facultad de Ciencias de la Educación de la Universidad Católica del Maule, UCM - Chile, Campus Nuestra Señora del Carmen, Carmen 684 Curicó. Dirección postal: Villa Pablo de Rokha psje. $6 \mathrm{~N}^{\circ} 2052$ Curicó.E-mail: tmendez@ucm.cl.
} 


\begin{abstract}
This article is a proposal for the teaching and learning of factoring quadratic polynomials, based Regine Douady's theoretical frameworks regarding the Tool-Object Dialectic. It highlights the role of combined mathematical frameworks as a means to access... "different formulations of a problem which, without necessarily being equivalent, allow a new approach to the difficulties encountered and the staging of tools and techniques that were not imposed in the first formulation." From this perspective, an axiomatic system is constructed in a figural context in which primary objects are defined, and relationships are established between them, leading to definitions and theorems of a mathematical theory within this framework. Rules are proposed for the operation of the primary objects and a game is described in which the factoring is operationalized using this framework. The game becomes the vehicle for understanding the difficult relations of the objects of factoring in the algebraic framework. This form of educational work develops cognitive and metacognitive skills in students who discover and describe the factorization properties, establish the algebraic relations between the coefficients of the polynomial and the factors that factored expression, developing a mathematical activity.
\end{abstract}

Keywords: Factoring. Figural Context. Categories of Factoring. Framework Game.

\title{
1 Introdución
}

Hay dos aspectos relevantes en las dificultades que presentan los alumnos al factorizar polinomios cuadráticos: primero, no comprenden que deben encontrar dos polinomios irreducibles, que al multiplicarlos den la expresión original y el segundo aspecto, encontrar dos números, que dependen de operaciones claramente establecidas, entre los coeficientes del polinomio y que permitan su factorización. Estas dos dificultades no pueden ser resueltas solo considerando el marco algebraico, como se evidencia en el análisis de los procedimientos y conocimientos que hay que poner en juego al factorizar.

El presente articulo propone la elaboración de un marco figural como sistema matemático, que permita dar sentido a los procedimientos que los alumnos realizan en él, sirviendo, este, de medio para comprender la factorización de polinomios cuadráticos. En esta teorización se identifican objetos, que permiten generar polinomios, se admiten axiomas y definiciones y se deducen propiedades, que son análogas a las propiedades de los polinomios del marco algebraico. Esta propuesta surge desde el enfoque didáctico, sobre la enseñanza de la matemática, la dialéctica herramienta - objeto y el juego de marcos matemáticos de Regine Douady, y constituye un ejemplo operacional de esta teoría. 
Un álgebra de polinomios tiene como objetos a los polinomios cuadráticos sobre un anillo, $\boldsymbol{Z}$ y - o sobre el anillo $\boldsymbol{Q}$, los axiomas de anillo y sus propiedades; análogamente en el marco figural existen objetos, que identificamos como figuras rectangulares, y reglas que son los axiomas, definiciones y propiedades, que permitirán la factorización.

La estructura de este artículo es la siguiente: Problemática, Estudio sobre las dificultades que los estudiantes presentan al factorizar polinomios cuadráticos, explicitación del marco figural y su naturaleza matemática y didáctica. Además expone los resultados de una experiencia reciente, utilizando este marco, con estudiantes de primer año de la carrera de Pedagogía General Básica con mención, de la Universidad Católica del Maule, finalmente algunas conclusiones

\section{Problemática}

El saber escolar considera que los polinomios cuadráticos $a_{2} x^{2}+a_{1} x+a_{0}$ son factorizables si existen $(x+\alpha)$ y $(x+\beta)$ polinomios irreducibles, tal que:

(1) $a_{2} x^{2}+a_{1} x+a_{0}=a_{2}(x+\alpha)(x+\beta)$,

$\alpha, \beta \in Q$, y $a_{2} \cdot(\alpha+\beta)=a_{1} ; a_{2} \cdot(\alpha \cdot \beta)=a_{0}$

Por ejemplo, dado $f(x)=2 x^{2}+3 x+1$ existen $p_{l}(x)=x+1 \quad y$ $p_{2}(x)=2 x+1$ irreducibles, tales que:

$$
f(x)=p_{1}(x) \cdot p_{2}(x)
$$

En efecto $2 x^{2}+3 x+1=(x+1) \cdot(2 x+1)$;

Bajo el marco algebraico se presentan fenómenos didácticos que no se pueden resolver con los objetos que viven en él.

Consideremos el polinomio $x^{2}-x-6$, un análisis de la factorización $x^{2}-x-6=(x-6)(x-1)$ realizada por un estudiante nos conduce a caracterizar el marco algebraico de la siguiente manera:

- Promueve este tipo de respuestas, que son incompletas y / o incorrectas

- Los estudiantes no se da cuenta si su respuesta es acertada o no, porque el marco no le permiten visualizar la reversibilidad de la operación

- Los estudiantes copian la estructura de la factorización pero no se dan 
cuenta y no comprenden las operaciones que han llevado a cabo.

Es decir en el marco algebraico existen dificultades persistentes de los alumnos, por lo que se revela insuficiente como único medio de enseñar este tema.

\section{Categorías de la Factorización}

Para explicar las dificultades de los estudiantes al factorizar, tomaremos como referencia las categorías de análisis de las nociones matemáticas en juego en la factorización de la tesis de magíster Dificultades en la práctica de la factorización y de las identidades notables (MÉNDEZ, 2001). Estas categorías intentan responder a la pregunta ¿qué conocimientos son necesarios para elegir el procedimiento que factoriza a un polinomio dado? Una conclusión de esta tesis es que las categorías revelan: procesos mentales que efectúan los estudiantes al factorizar y conocimientos matemáticos a poner en juego, estos dos son procesos indisociables.

Las categorías destacan tareas y subtareas, en los procedimientos efectuados por los alumnos, ellas ponen en evidencia la relación entre los aspectos matemáticos y cognitivos, implícitas en su ejecución.

Las tareas se definen como procedimientos a efectuar relacionados con conceptos y propiedades matemáticas, y las subtareas corresponden a un desglose más fino de la tarea propuesta por la complejidad del ejercicio.

En cada categoría hay una tarea matemática a efectuar, factorizar, y una tarea cognitiva que tiene que ver con el procedimiento a utilizar en la factorización. ¿Recurrirá a la fórmula de factorización? ó reconocerá la suma por diferencia (cuadrado de binomio,...) e identificará los términos de su ejercicio con los del enunciado de la fórmula (tarea cognitiva y matemática a la vez).

Hay cambios de variables implícitos en estos procedimientos que dificultan las prácticas de los alumnos.

\subsection{Categoría factorizar un monomio}

Factorizar el monomio $3 a x^{2} y$

Podemos considerar este ejemplo como un monomio en varias variables en que algunos ejemplos de su factorización son: 


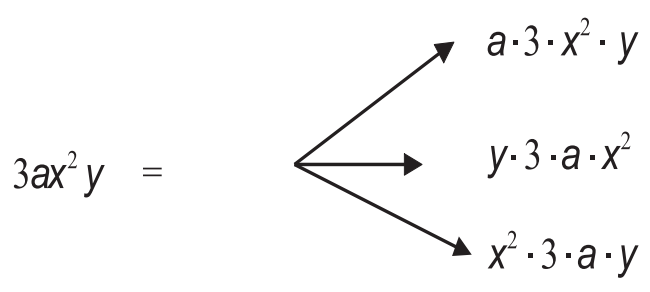

Esta categoría destaca la tarea de reconocer que un monomio es un producto de factores, y que para reescribirlo, se utiliza la propiedad conmutativa.

El alumno que no logra esta abstracción tiene dificultades en comprender que la expresión $x^{2}+4 x+4$ se descompone en factores de distinta naturaleza, claro está, que en el ejemplo anterior.

\subsection{Categoría Factorizar un polinomio cuadrático ordenado}

Esta categoría se relaciona con el teorema de la factorización única, distinguiéndose las tareas siguientes:

3.2.1 Factorizar trinomio cuadrático ordenado, implica tareas relacionadas con la definición de polinomios divisibles, y con el teorema de la factorización única. Surgen las siguientes subtareas: si el polinomio es de la forma $x^{2}+b x+c$ ó $a x^{2}$ $+b x+c ;$ con $a \neq 0 ; a \neq 1$

i) Sea $x^{2}+b x+c$, la tarea consiste en determinar números: $\alpha$ y $\beta$, tales que:

$$
\alpha+\beta=b \text { у } \alpha \cdot \beta=c,
$$

Para formar polinomios irreducibles $(x+\alpha)$ y $(x+\beta)$ que factoricen, utilizando la fórmula (1),

$$
x^{2}+b x+c=(x+\alpha) \cdot(x+\beta)
$$

Por ejemplo los números que factorizan $x^{2}-x-30$ son: $\alpha=5$ y $\beta=-6$, ya que $\alpha+\beta=-1$ y $\alpha \cdot \beta=-30$, y su factorización es:

$$
x^{2}-x-30=(x+5) \cdot(x-6)
$$


ii) Sea el polinomio $x 2+b x+c$, la tarea consiste en determinar $\alpha y \beta$, tales que:

$$
a \cdot(\alpha+\beta)=b \quad \text { y } \quad a \cdot(\alpha \cdot \beta)=c
$$

Para formar polinomios irreducibles $(x+\alpha)$ y $(x+\beta)$ que factoricen de acuerdo a la fórmula (1),

$$
a x^{2}+b x+c=a \cdot(x+\alpha) \cdot(x+\beta)
$$

Por ejemplo los números, $\alpha=-5$ y $\quad \beta=\frac{3}{2}$ que factorizan $2 x^{2}-7 x-15$ son tales que: $2 \cdot\left(-5+\frac{3}{2}\right)=-7 \quad$ y $2 \cdot\left(-5 \cdot \frac{3}{2}\right)=-15$ su factorización es: $2 x^{2}-7 x-15=2 \cdot(x-5) \cdot\left(x+\frac{3}{2}\right)=(x-5)(2 x+3)$ (ver fórmula (1))

3.2.2 Factorizar trinomio ordenado cuadrado perfecto, de la forma $a^{2} x^{2}+2 a b x+b^{2}$

Aquí la tarea consiste en:

- Reconocer el desarrollo del cuadrado del binomio.

Por ejemplo si se pide factorizar:
i) $x^{2}+4 x+4$
ii) $4 x^{2}+2 x+\frac{1}{4}$

El alumno tiene que reconocer (es una tarea cognitiva) en cada trinomio, el desarrollo de un cuadrado de binomio y factorizarlo, ó utilizando las reglas de la tarea 3.2.1, buscar dos números que multiplicados entre sí, y por $a^{2}$ den como resultado $b^{2}$ y cuya suma por $a^{2}$ de $2 a b$, para luego sustituirlos en la fórmula. En esta tarea existe un cambio de variable implícito, que la dificulta.

$$
a^{2}\left(x+\frac{b}{a}\right)\left(x+\frac{b}{a}\right)=(a x+b)^{2} .
$$

En el primer ejemplo, $a^{2}=1, b^{2}=4$ y $2 a b=4$ así los números son 
iguales a dos y su factorización es $1 \cdot(x+2) \cdot(x+2)=(x+2)^{2}$

En el segundo ejemplo $b^{2}=\frac{1}{4}$ y $a^{2}=4$ y $2 a b=2$, así los números son iguales a $\frac{1}{4}$

y su factorización es $4 \cdot\left(x+\frac{1}{4}\right) \cdot\left(x+\frac{1}{4}\right)=4\left(\frac{4 x+1}{4}\right)^{2}=\left(2 x+\frac{1}{2}\right)^{2}$.

3.2.3 Factorizar el polinomio utilizando propiedades de anillo.

Por ejemplo, al factorizar $5 x^{2}+3 x-14$, utilizaremos las propiedades: descomposición aditiva y las de anillo.

$$
\begin{array}{ll}
5 x^{2}+3 x-14 & \text { Descomponer } 3 x=10 x-7 x \text { y reemplazar } \\
5 x^{2}+10 x-7 x-14 & \text { Asociar y factorizar por } 5 x \text { y } 7 \\
\left(5 x^{2}+10 x\right)-(7 x+14) & \\
5 x(x+2)-7(x+2) & \text { Factorizar por }(x+2) \\
(x+2)(5 x-7) &
\end{array}
$$

Así los números que factorizan al polinomio son $\alpha=2$ y $\beta=\frac{-7}{5}$.

y $5 \cdot(x+2) \cdot\left(x-\frac{7}{5}\right)=(x+2) \cdot(5 x-7)$ es su factorización.

Este análisis revela que la $4^{\text {a }}$ categoría es más compleja y completa y requiere desglosarla en tareas más específicas.

\subsection{Categoría Factorización de un polinomio}

Análisis de la factorización de un binomio en monomio por binomio y en binomio por binomio.

En esta categoría se pueden reconocer la existencia de dos propiedades 
algebraicas: la definición de polinomio irreducible, ejemplo 3.3.1i y el teorema de la factorización única, ejemplos 3.3.1ii y siguientes.

3.3.1. Factorizar binomios en monomio por binomio:

Por ejemplo:

i) $3 x+6=3 x+3 \cdot 2=3(x+2)$

ii) $\quad 6 a x^{2}-2 a^{2} x=2 a x \cdot 3 x-2 a x \cdot a=2 a x(3 x-a)$

- La tarea requiere buscar factor común en los términos del binomio. $a x^{2}+b x=x \cdot a x+x \cdot b=x \cdot(a x+b)$

3.3.2 Factorizar binomio diferencia de cuadrados en producto de binomios:

Esta tarea se desglosa en tres subtareas:

- Reconocer suma por diferencia

- Hacer cambio de variable implícito.

- Utilizar reglas de la tarea 3.2.1

Por ejemplo:

3.3.3 Al factorizar $x^{2}-16=x^{2}-4^{2}=(x+4) \cdot(x-4)$, el alumno debe reconocer los términos de una diferencia de cuadrados, y factorizarla en suma por diferencia

3.3.4 Al factorizar $x^{4}-16=\left(x^{2}+4\right) \cdot\left(x^{2}-4\right)$

La tarea se desglosa en las siguientes etapas:

Cambio de variable, implícito

Sea $y=x^{2}$, entonces $y^{2}=x^{4}$ y $4^{2}=16$, así: $x^{4}-16=y^{2}-16$

Reconocer términos de la diferencia de cuadrados se factorizar: $(y+4)(y-4)$

Cambio de variable:

$$
(y+4)(y-4)=\left(x^{2}-4\right) \cdot\left(x^{2}+4\right)
$$


La tarea exige cambiar de variable para reconocer los términos de una diferencia de cuadrados y factorizarla en suma por diferencia.

Todos estos procedimientos, justificados por la lógica del trabajo matemático, dificultan las decisiones que los alumnos toman al resolver estos ejercicios.

Dada la complejidad de estos procesos, este articulo propone la elaboración del marco figural como herramienta didáctica que permita al estudiante solucionar los problemas de factorización que el sistema educativo solicita en el nivel correspondiente, pero además este nuevo marco permite construir una teoría axiomática, descubrir y enunciar propiedades de la factorización en él y comprender el mecanismo algebraico de la factorización, es decir el estudiante desarrolla una actividad matemática.

A continuación se explicitan los objetos de este marco.

\section{Marco Figural}

Consideremos los siguientes objetos primarios: figuras rectangulares y un juego cuyas reglas permiten la construcción de polinomios cuadráticos y su factorización. Estos objetos son la expresión figural de los términos, y, de un polinomio cuadrático ${ }^{1}$.

Se define lo siguiente.

- Figuras rectangulares rojas de lados $\boldsymbol{x}$ y $\boldsymbol{x}$ que se anotan $x^{2}\left(\mathrm{o} R_{\mathrm{xx}}\right)$

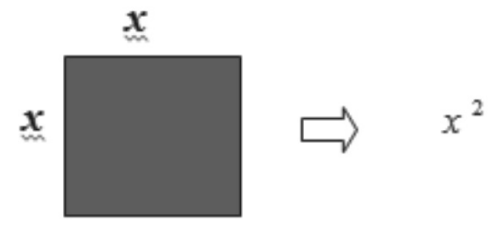

- Figuras rectangulares azules, opuestas a las rojas que se anotan $-x^{2}$ (ó $R_{\mathrm{XX}}$ )

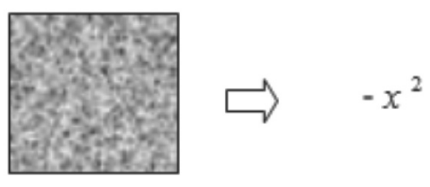

\footnotetext{
${ }^{1}$ Por razones de impresión, cuando hablemos de figuras rectangulares rojas utilizaremos figuras plomas lisas, como estas y para las figuras rectangulares azules se utilizará el color plomo jaspeado
} 
- Figuras rectangulares rojas de lados $\boldsymbol{x}$ y 1 , que se anotan $x\left(\mathrm{o} R_{\mathrm{x} 1}\right)$.

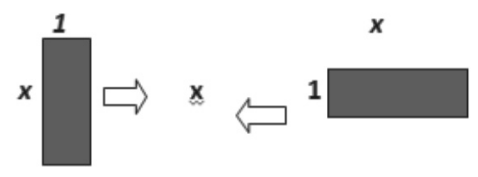

- Figuras rectangulares azules, opuestos a las rojas, de lados $x$ y -1 , que se anotan - $x\left(\right.$ ó $\left.-R_{\mathrm{x} 1}\right)$.

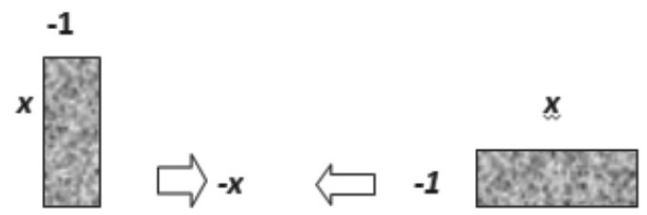

- Figuras rectangulares rojas de lados 1 y $\boldsymbol{1}$ que se anotan 1 (o $R_{11}$ )

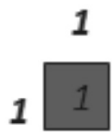

- Figuras rectangulares azules, de lados 1 y -1 , opuestas a las rojas, que se anotan -1 (ó $\left.-R_{11}\right)$

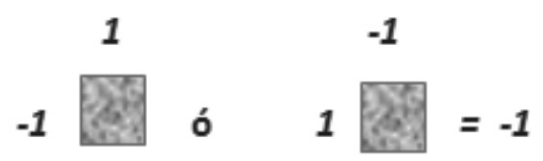

Observación:

Las figuras de color rojas representan términos algebraicos positivos y las de color azules sus opuestos, para efectos de factorizar, consideraremos que el lado que comparten los objetos primarios con textura, $R_{\mathrm{x} 1}$ y $R_{11}$, es -1

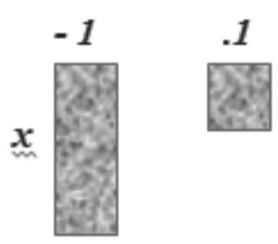


Consideremos el juego siguiente:

Armar figuras rectangulares que combinan objetos primarios según las siguientes reglas.

\subsection{Reglas Relacionadas con la unión de las piezas:}

Regla 1: Las figuras sólo se yuxtaponen

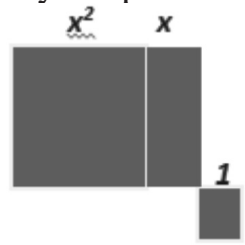

Regla 2: Figuras distintas solo se unen por lados en común, si existen, o por sus vértices si no existen.
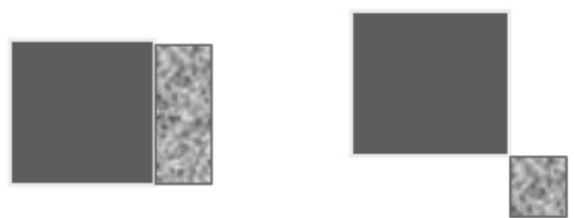

Recomendación: Yuxtaponer primero las figuras rectangulares de lado $\boldsymbol{x}$ y $\boldsymbol{x}$ $\left(R_{\mathrm{xx}}\right)$, luego las de lado 1 y $1\left(R_{11}\right)$ y finalmente distribuir las figuras de lados $\boldsymbol{x}$ y $\boldsymbol{I}\left(R_{\mathrm{x} 1}\right)$ y o $\boldsymbol{x}$ y $-\boldsymbol{I}\left(-R_{\mathrm{x} 1}\right)$ en forma vertical y horizontal.

\subsection{Reglas relacionadas con la factorización de polinomios cuadráticos}

Definición 4.2.1: Factorizar un polinomio cuadrático, consiste en construir una figura rectangular, con los objetos primarios, tal que el producto de sus dimensiones, largo y ancho, es su factorización.

Al ubicar los objetos primarios se cumple que:

Regla 3a: Si una figura rectangular se forma con objetos primarios de lado 1 rojas, $R_{11}$ entonces las figuras de lados $\boldsymbol{x}$ y $\boldsymbol{1}$, verticales y horizontales son del mismo color entre sí (todos rojas o todos azules)

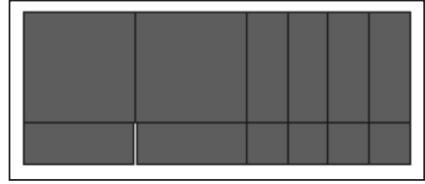

Figura 1

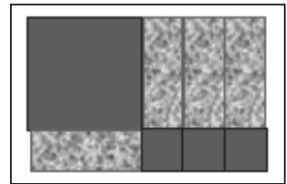

Figura 2 
Regla $3 b$ : Si un figura rectangular se forma con objetos primarios de lado 1 azules, $-R_{11}$,entonces las figuras verticales y horizontales, de lados $\boldsymbol{x}$ y 1 , son de colores diferentes.

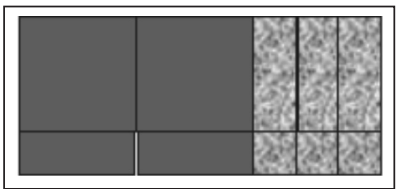

Figura 3

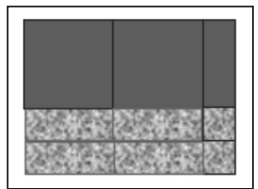

Figura 4

Regla 4: Un polinomio cuadrático es factorizable, si y solo si:

Regla 4a: Con los objetos primarios se puede formar una figura rectangular según las reglas 1,2 y $3 a$ anteriores

Regla 4b: Al formar la figura se produce un defecto en número par de objetos

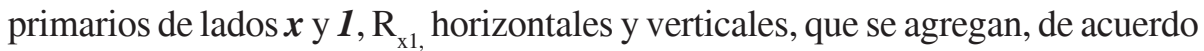
a la regla $3 b$

Regla 5a: Objetos primarios congruentes de igual color se suman, entre sí. Regla 5b: Objetos primarios congruentes de distinto color se restan, entre sí. Veamos el siguiente ejemplo, en el que se aplican estas reglas:

Ejemplo 1:

Sea el polinomio:

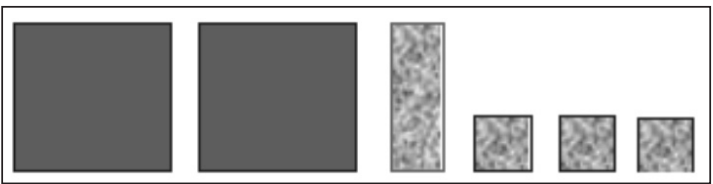

Figura 5

Su rectangularización requiere utilizar la regla $4 b$

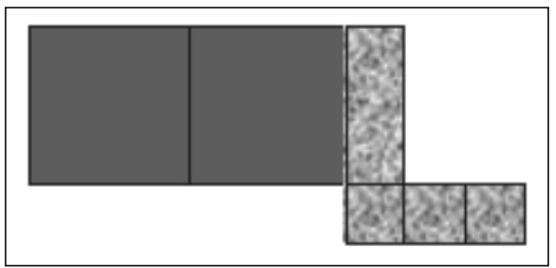

Figura 6 
Agregando dos parejas de objetos $R_{11} \mathrm{y}-\mathrm{R}_{\mathrm{x} 1}$ que se disponen horizontal y verticalmente, para completar la figura. Esto $\mathrm{es}^{1}$ :

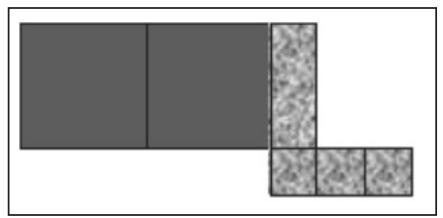

Figura 7

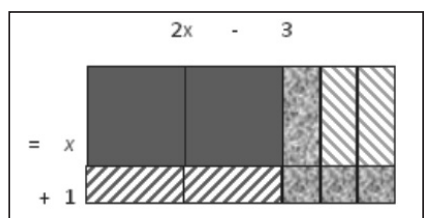

Figura 8

Se observa que las dimensiones de sus lados son: $2 x-3$ y $x+1$, de modo que su factorización es $2 x^{2}-x-3=(2 x-3) \cdot(x+1)$

\section{Marco Teórico}

Este trabajo considera el enfoque didáctico de Regine Douady (1986, p. 6), quien sostiene que "Se puede construir efectivamente conocimientos matemáticos articulando marcos apropiados de representación de los objetos matemáticos, siempre que los problemas respondan a ciertas condiciones."

Esta articulación de marcos matemáticos es lo que Regine Douady (1986) ha llamado juego de marcos, que consiste en proponer a los estudiantes una situación problema que no pueden resolver completamente, en el marco en el que esta formulado, debido a la debilidad de sus conocimientos. Entonces es necesario poner en funcionamiento otro marco que permita acceder a una representación equivalente de los objetos matemáticos que necesita operacionalizar. Este marco nuevo se vuelve una herramienta para resolver el problema y, además, permite comprender las complejidades del marco de partida, ya que los conocimientos que se ponen en juego en el marco nuevo transitan de uno a otro produciendo efectos favorables para el aprendizaje y comprensión de este concepto.

Veamos el siguiente ejemplo:

Ejemplo 2:

En la factoriación de $3 x^{2}-7 x-6$ los conceptos matemáticos, antiguos, son puestos en obra como herramientas explícitas para resolver al menos parcialmente el problema.

Sin embrago un alto porcentaje de alumnos responde incompleta e

\footnotetext{
${ }^{1}$ En las figuras 8, 10 y 11 se han insertado figuras auxiliares achuradas, mediantes líneas diagonales en los respectivos colores y lugares, de acuerdo a las reglas establecidas, para rectangularizar y factorizar.
} 
incorrectamente, en el marco algebraico, por ejemplo $3 x^{2}-7 x-6=(3 x-3)(x$ -2 ), se observa una factorización parcial del polinomio, producida por un uso inadecuado de la factorización como objeto matemático, y de conocimientos insuficientes de los alumnos.

El profesor intenciona la búsqueda de un nuevo implícito, definiendo objetos primarios en un marco figural, que permita representar los términos de un, polinomio cuadrático, como al mismo, a través de figuras rectangulares, cuyas dimensiones dan cuenta de su factorización.

Utilizando el marco figural, la representación del polinomio es:
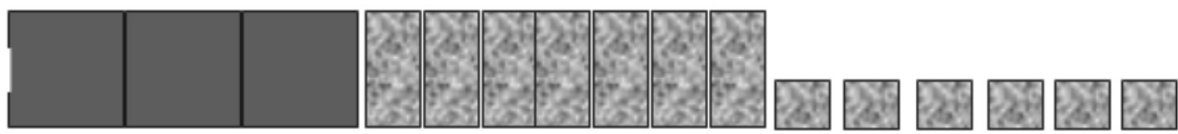

Distribuyendo los objetos primarios de acuerdo a las reglas, se obtiene:

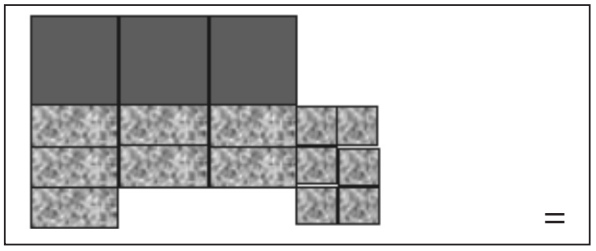

Figura 9

Según la regla $3 b$, hay que agregar dos parejas de objetos primarios una roja y otra azul, $R_{\mathrm{x} 1} \mathrm{y}-R_{\mathrm{x} 1}$ que se disponen de la siguiente manera, para completar la rectangularización y obtener la factorización:

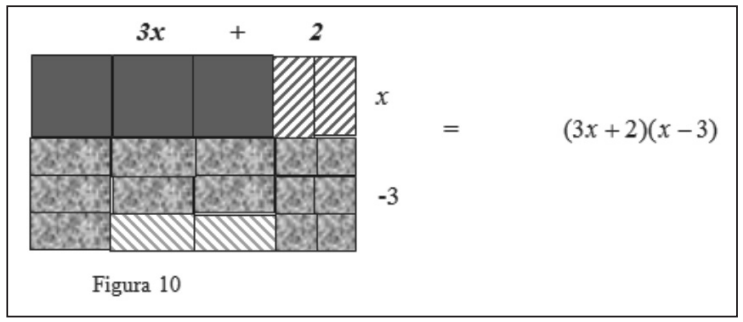

Figura 10

En este ejemplo el medio es la representación del polinomio en el marco figural, cuya distribución en rectángulo permite identificar el producto de sus dimensiones con la factorización buscada. La comprobación de esta operación se da en el registro figural y/o algebraico. El concepto que surge en el trabajo 
del alumno, y desconocido como objeto, es la factorización que, implícitamente, el medio figural permite construir en este marco para darle estatus de objeto de estudio para el alumno.

Entonces en el juego de marcos los alumnos son enfrentados a un problema de factorización, formulado en un marco algebraico. Teniendo en cuenta sus conocimientos y sus procedimientos, el análisis que hacen del problema los lleva a traducir a un marco figural, interpretando en él las preguntas planteadas. Ante las dificultades presentadas en algunos alumnos, se vuelve a reforzar el sentido de las reglas, en el marco figural y su relación con las operaciones en el marco algebraico, a fin de interpretar la información en este nuevo marco.

Se perciben, aquí, las condiciones que deben satisfacer las situaciones problemas, en el juego de marcos: la resolución del problema planteado debe pasar, obligatoriamente, por la utilización como medio de él o los conceptos avistados. Los conceptos son, así, introducidos por su funcionamiento.

La resolución del problema planteado debe hacer intervenir el saber enseñado (saber escolar, lo que se enseña) o aquel del cual se quiere observar los procesos de adquisición en marcos diferentes: el marco figural en nuestro caso. El motor de la acción de los alumnos es el hecho que los conocimientos que tienen rasgos de conceptos a adquirir no son manejados de la misma manera en los distintos marcos matemáticos. Los conocimientos son más amplios en uno de los marcos y esto les da las ideas de procedimientos a verificar.

En una sucesión de situaciones debe haber lo que ha llamado dialéctica medio-objeto, el concepto interviene primero como medio implícito en la fase de construcción de la noción, luego él es reconocido como objeto después de la fase de institucionalización. Él podrá, desde entonces, intervenir como medio explícito en la construcción de otra noción.

Para Régine Douady, el saber avistado es considerado como adquirido, cuando el concepto es utilizado como medio explícito en problemas donde no es evidente su intervención aunque lo haga en forma implícita y/o explícita.

La situación debe anclarse en saberes ya adquiridos, con el fin de permitir a los alumnos tener estrategias de base para poder enfrentar los problemas. Ella debe ser suficientemente abierta para que los alumnos puedan ensayar diversos procedimientos y puedan elegir libremente aquellos que les parezcan los más adecuados, pero no demasiado abierta ya que impediría a los alumnos introducirse en los campos conceptuales anexos o se desviarían ensayando otros procedimientos. 
El marco figural permite decidir si el polinomio es factorizable o no, como veremos en el siguiente ejemplo desarrollado en ambos marcos:

Ejemplo 3: Sea el polinomio $f(x)=x^{2}+2, f(x)$ es irreducible en $Q[x]$, pues no existen polinomios, en $Q[x], h(x)$ y $q(x)$, tales que su producto sea $x^{2}+2$

En efecto si existieran tales $h(x)$ y $q(x)$, se tendría que: $x^{2}+2=2 \cdot\left(\frac{x^{2}}{2}+1\right)$, en que $h(x)$ es constante ó $x^{2}+2=x \cdot\left(x+\frac{2}{x}\right)$, en que $q(x) \notin Q[x]$

En el marco figural, sea el polinomio:
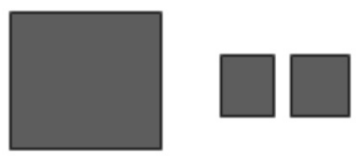

Al aplicar las reglas, el polinomio no cumple con la regla 4, es decir no se puede rectangulartizar porque se produce una diferencia impar de figuras $\mathrm{R}_{\mathrm{x} 1}$, luego $f(x)$ es irreducible.

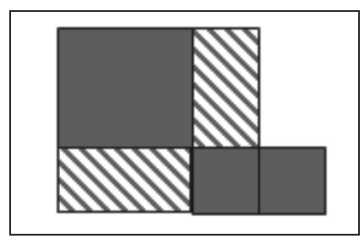

Figura 11 - polinomio irreducible

\section{Naturaleza del marco Figural}

El marco figural funciona en forma análoga a un marco matemático, en efecto, se proponen objetos, definiciones y teoremas relacionados con las reglas establecidas anteriormente.

Definición: Un polinomio cuadrático es una yuxtaposición de figuras rectangulares con las convenciones siguientes:

$a$ figuras rectangulares de lado $x$ y $x$, asociadas con términos cuadráticos, $b$ figuras rectangulares de largo $x$ y ancho $l$ (uno), asociadas a los términos lineales y $c$ figuras rectangulares de lados 1 y 1 asociadas a términos constantes.

Los siguientes son polinomios cuadráticos en el marco figural 
Ejemplo 4:

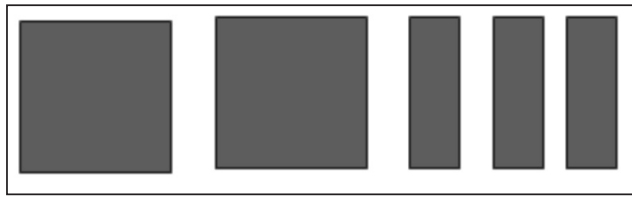

Figura 12 - polinomio $2 x^{2}+3 x$

Ejemplo 5:

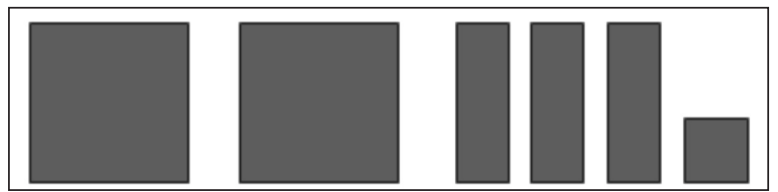

Figura 13 - polinomio $2 x^{2}+3 x+1$

Ejemplo 6:

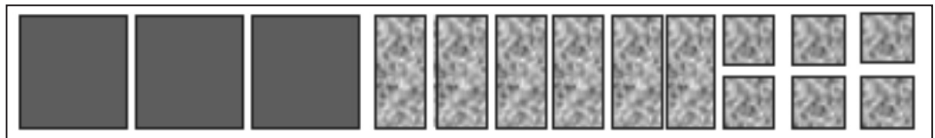

Figura 14 - polinomio $3 x^{2}-6 x-6$

En este marco es posible definir la suma y producto de polinomios como sigue:

\subsection{Suma de polinomios cuadráticos en el marco figural}

Dados dos polinomios cuadráticos, en el marco figural, su suma viene dada por la suma de las figuras rectangulares congruentes entre sí.

Ejemplo 7: aplicando la regla $5 a$ y $5 b$ a la suma de estos polinomios se obtiene:

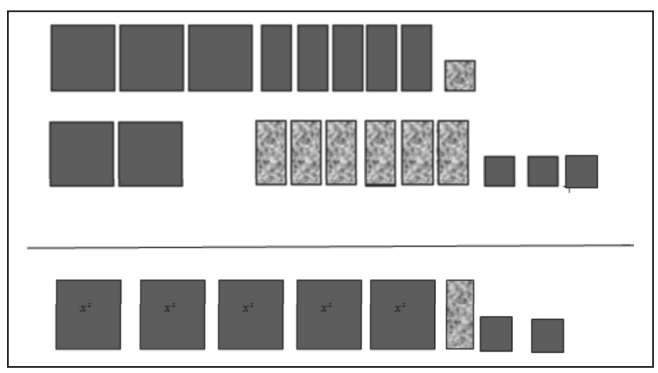

Figura 15 - suma de polinomios 
Que es la representación figural de:

$$
\left(3 x^{2}+5 x-1\right)+\left(2 x^{2}-6 x+3\right)=\left(3 x^{2}+2 x^{2}\right)+(5 x+-6 x)+(-1+3)=5 x^{2}-x+2
$$

\subsection{Multiplicación de binomios lineales en el marco figural}

Es la figura rectangular cuyos lados son los factores a multiplicar. Su producto es la suma de las figuras rectangulares que se determinan, utilizando la regla 5 .

Ejemplo 8: la siguiente figura

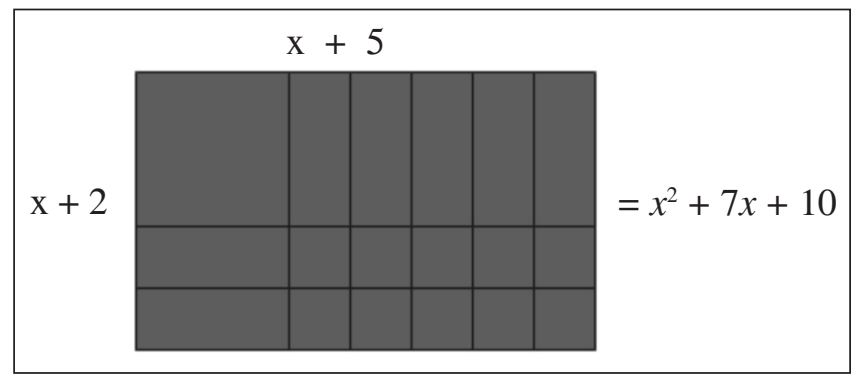

Figura 16 -Representa la multiplicación: $(x+5) \cdot(x+2)=x^{2}+7 x+10$

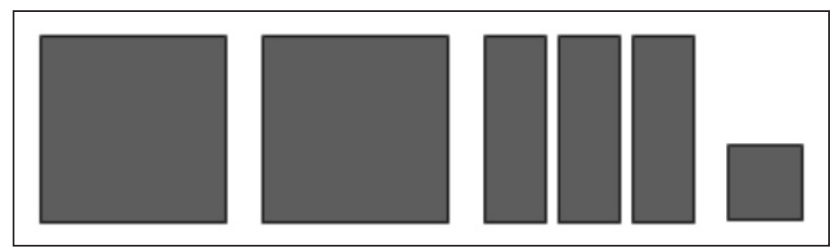

Figura 17

una distribución de sus objetos en:

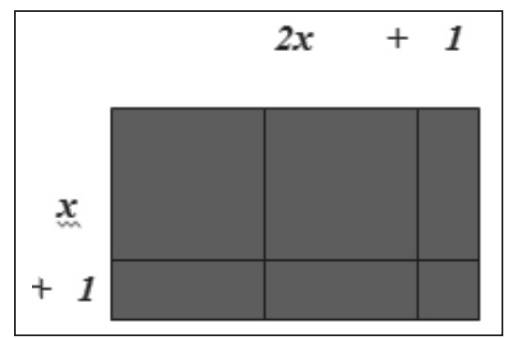

Figura 18 
nos indica que $f(x)$ es divisible en $h(x)((x+1))$, ya que existe $c(x)((2 x+1))$ tal que

$$
f(x)=h(x) \cdot c(x)
$$

La representación en el marco algebraico, de este ejemplo es:

$$
2 \times 2+3 x+1=(2 x+1)(x+1) \text {. }
$$

Definición 6.5: $f(x)$ es un polinomio irreducible, si cada vez que se escriba como producto de dos polinomios $f(x)=h(x) \cdot q(x)$ entonces uno de ellos, $h(x)$ o $q(x)$ es una constante.

Por ejemplo, dado el polinomio $x^{2}+1$, algebraicamente se demuestra su irreductibilidad en $Q[x]$, por razonamiento indirecto, y además se comprueba considerando que los ceros de este polinomio no son reales (racionales).

En el marco figural, el polinomio $\square$ es irreducible, pues utilizando la regla $5 b$, la figura se puede rectangularizar, agregando una pareja de objetos primarios $R_{x 1} \mathrm{y}-R_{x 1}$, pero la regla $3 a$ impide este procedimiento, al establecer que si es rojo, entonces los objetos $R_{x 1}$ son del mismo color. Luego el polinomio es irreducible.

\section{Resultados}

Recientemente se realizo un pretest a 16 estudiantes de primer año de la carrera de pedagogía general básica con mención, de la universidad Católica del Maule, (que ya habían estudiado, en la enseñanza media, este tema) con intención de elegir la mención matemática.

El pretest consistía en una batería de ejercicios, propuestos en el registro algebraico, en que los estudiantes debían demostrar su experticia en el procedimiento de factorización de polinomios de la forma $a x^{2}+b x+c$ y $x^{2}+b x$ $+c$, en operaciones con fracciones algebraicas en las que intervenían esos tipos de polinomios, justificando los procedimientos puestos en obra, y un ítem en que dado un conjunto de figuras rectangulares, como las del marco figural se pedía organizarlas para formar un cuadrado que representara la factorización $(\mathrm{x}+6)^{2}$

Nueve de ellos obtuvieron puntuaciones por debajo del $60 \%$ de exigencia aprobatoria.

Un análisis de las respuestas de los estudiantes da cuenta de las debilidades de los conocimientos de los estudiantes cuando factorizan en el marco algebraico. Los procedimientos mas recurrentes son: los estudiantes copian un 
modelo de paréntesis de la factorización; factorizan buscando factor común monomio; reducen términos semejantes; simplifican sumandos del polinomio del mismo grado y semejantes en las fracciones algebraicas, entre otros procedimientos.

Nueve de los 16 estudiantes se sometieron a un tratamiento de aproximadamente 8 horas, que consistió en:

- Definir los objetos del marco figural y sus reglas, utilizarlo en la factorización de polinomios de la forma $a x^{2}+b x+c$ y $x^{2}+b x+c$, efectuar la articular ambos marcos matemáticos, descubrir la regla algebraica de la factorización del polinomio, establecer la existencia de polinomios no factorizables en el marco figural y comprender la dificultad de este procedimiento en el marco algebraico.

- Respecto de los polinomios de la forma $a x^{2}+b x+c$, su factorización en el marco figural fue una herramienta importante para comprender su factorización algebraica y su complejidad, al momento de institucionalizarla.

Posteriormente se les aplico un postest que se evalúo al mismo porcentaje de exigencia, y sus puntuaciones se ubicaron en el cuarto superior del puntaje total, además el modelo implementado tuvo buena aceptación y evaluación de parte de los participantes. De las nueve personas en tratamiento, cinco obtuvieron primero fracaso en el pretest y posteriormente éxito en el postest.

Esta última experiencia muestra que, en el caso de la factorización de polinomios cuadráticos, el marco figural contribuye a superar las dificultades de muchos estudiantes que ven fracasados sus intentos en el marco algebraico, y, por otra parte, es un trabajo que los alumnos desempeñan percibiendo progresos en la tarea que se les presenta y a la vez deducen propiedades de la factorización, de acuerdo a las reglas establecidas, que en el modelo algebraico son teoremas

\section{Conclusión}

El juego de marcos se constituye en un modelo eficaz para la enseñanza - aprendizaje de la factorización de polinomios cuadráticos que se operacionaliza a través del marco figural, desarrollando habilidades cognitivas en los estudiantes, en oposición al marco algebraico que ofrece un trabajo estático cuyos efectos son bien conocidos por profesores e investigadores.

Estas habilidades cognitivas están relacionadas con estructuras lógico matemática como la reversibilidad, ya que el polinomio se puede armar y desarmar, 
se puede hasta completar si es necesario con la finalidad de rectangularizarlo para hallar su factorización. Existe búsqueda de regularidades que conducen a obtener una formula para la factorización de polinomios de la forma $x^{2}+b x+c$ primero y luego de $a x^{2}+b x+c$.

Existen niveles de abstracción diferentes, primero la acción, en que el alumno utiliza el marco figural solo con la finalidad de factorizar, posteriormente formulación de regularidades y descubrimiento de una formula algebraica.

Sus características son:

Los términos de un polinomio cuadrático, son representados por figuras rectangulares definidas de acuerdo a los términos de un polinomio algebraico de segundo grado, y su factorización se obtiene organizando las figuras en cuadrado o rectángulo, cuyas dimensiones dan cuenta de la factorización del polinomio.

El marco figural es de naturaleza matemática pero tiene propiedades didácticas que permiten la apropiación con sentido del concepto de factorización.

Permite la factorización de todos los polinomios cuadráticos factorizables en $Q[x]$, del marco algebraico, y sus reglas permiten justificar que existen polinomios cuadráticos en $Q[x]$ que no se pueden factorizar porque las transgreden.

Los alumnos transitan factorizando en el marco figural comprendiendo las relaciones que se cumplen en el marco algebraico.

Este marco se vuelve una herramienta ya que los conocimientos que se ponen en juego en él, transitan de uno a otro marco operacionalizando el juego de marcos cuyos efectos son favorables para el aprendizaje y comprensión de los conceptos, como hemos visto en los diferentes ejemplos.

\section{Referencias}

DOUADY, R. Jeux de cadres et dialectique outil-objet. Recherche en Didactique des Mathématiques - La Pensée Sauvage. Grenoble, v.7, n.2, p.5-32, 1986.

DOUADY, R. Relación Enseñanza - Aprendizaje. Dialéctica Instrumento-Objeto, Juego de Marcos. Cuaderno de didáctica de la matemática. n.3. Disponible en: <http:// es.scribd.com/doc/3930820/PMAT-Douady-Unidad-2>. Acceso en: 12 jun. 2011.

MÉNDEZ, T. Dificultades en la práctica de la factorización y productos notables. 2001. 108f. Tesis (Magister en Enseñanza de las Ciencias mención Didáctica de la matemática) - Universidad Católica de Valparaíso, Valparaíso, 2001. 
ANILLO DE POLINOMIOS. Disponible en: <www.saber.ula.ve/bitstream/123456789/ 15888/2/anillos_polinomio.pdf>. Acceso en: 04 abr. 2010.

Submetido em Agosto de 2011. Aprovado em Fevereiro de 2012. 